\section{$\underset{\substack{\text { hommes } \\ \text { \& migrations }}}{ }$}

\section{Hommes \& migrations}

Revue française de référence sur les dynamiques

migratoires

$1306 \mid 2014$

Ecriture et migration

\title{
May in the summer
}

Film jordanien français 2013 de Cherien Dabis

\section{Anaïs Vincent}

\section{Q OpenEdition \\ 1 Journals}

\section{Édition électronique}

URL : http://journals.openedition.org/hommesmigrations/2865

DOI : 10.4000/hommesmigrations.2865

ISSN : 2262-3353

\section{Éditeur}

Musée national de l'histoire de l'immigration

\section{Édition imprimée}

Date de publication : 1 avril 2014

Pagination : 161-162

ISBN : 978-2-919040-27-8

ISSN : $1142-852 X$

\section{Référence électronique}

Anaïs Vincent, "May in the summer», Hommes \& migrations [En ligne], 1306 | 2014, mis en ligne le 06 août 2014, consulté le 22 septembre 2020. URL : http://journals.openedition.org/hommesmigrations/ 2865 ; DOI : https://doi.org/10.4000/hommesmigrations.2865 


\section{FILMS}

Julien Aouidad

C'est comme ça. Histoires

d'Angevins venus d'ailleurs de 1960 à 1980

Documentaire français (2012)

C'est comme ça. Histoires "Je les vois comme des étrangers. Ils ne sont pas nés ici. Ils ont un accent..." Ces mots de petits-enfants de travailleurs immigrés ouvrent C'est comme ça. Histoires d'Angevins venus d'ailleurs de 1960 à 1980. Eux sont français et expérimentent "ces limites séparant juridiquement des nationaux et des étrangers et ces limites entre catégories sociales racialisées héritées d'une double histoire de la colonisation et de l'immigration distinguant des individus et des groupes sur des indices variables de couleur d'origine, de culture, voire de religion". Mais, en effet, c'est comme ça.

Comme son titre l'indique, C'est comme ça. Histoires d'Angevins venus d'ailleurs de 1960 à 1980, réalisé par Julien Aouidad à l'initiative de l'association Histoire et mémoire d'immigration en Anjou, a un ancrage local. Celle de la ville d'Angers, dont il retrace l'histoire de l'immigration à travers les souvenirs de ses habitants venus d'ailleurs. Ainsi, alors qu'au niveau national se tarissent lentement les flux migratoires à destination de la France, les trois décennies ici évoquées (années 1960, 1970 et 1980) se caractérisent par des vagues tardives d'immigration de Turcs, d'Algériens, de Marocains, de Tunisiens, puis d'Afri- cains du sud du Sahara. Ces populations nouvelles rejoignent d'autres immigrés venus essentiellement d'Europe (Espagne, Italie, Pologne, Belgique, Portugal et Russie qui, depuis plusieurs siècles, marquent la région. C'est comme ça montre la diversité des origines et des parcours des immigrations angevines.

Assis sur le conduit d'aération de la mine située à 480 mètres de profondeur où il a travaillé en arrivant en France en 1966, Manuel raconte sa fuite du fascisme à pied et en stop. Des années plus tôt, le traité de Rome avait institué la Communautééconomique européenne. Mais son pays d'origine, le Portugal, ne fait pas encore partie des signataires, il est donc un clandestin. Et pourtant, "c'était facile comme tout", dit-il.

La France a alors besoin d'"une maind'œuvre abondante bon marché et qu'on régularise quand elle est sur place". Elle se tourne donc aussi vers les anciens territoires coloniaux et signe des accords de recrutement de travailleurs locaux avec l'Algérie, le Maroc, la Tunisie et la Mauritanie.

Les travailleurs migrants s'engagent dans l'aventure migratoire avec, sinon une certaine insouciance, du moins une vision idéalisée de la France. Arrivé d'Aid-Said au Maroc en 1972, Moulay Abderramahne pensait devenir "riche et rendre la famille riche au pays". Puis il s'est rendu compte qu'"on ne gagne pas de fortune", au contraire. Conducteurs d'engins, cheminots ou mineurs immigrés découvrent la pénibilité du travail, les logements insalubres, les discriminations et le racisme. Mohamed, lui, débarque en 1963 sur le quai de la 
gare d'Angers, qui deviendra son lieu de travail et son domicile : On habitait sur la voie, on travaillait sur la voie, on mangeait sur la voie et on couchait sur la voie." Les immigrés découvrent aussi la solidarité. Abdelmalek Sayad remarquait à cet égard: "Chaque nouvelle vague d'émigrés en France y trouvait déjà établie une communauté formée d'immigrés plus anciens à laquelle elle pouvait s'agréger." Ce réseau bien implanté envoie des contrats de travail aux candidats à l'émigration dans le pays d'origine et prend en charge les nouveaux arrivants. Et peu à peu avec l'appui des syndicats, les travailleurs immigrés obtiennent les mêmes droits que les travailleurs français. À partir des années 1970, les travailleurs immigrés sont rejoints par leurs familles. Le film s'attache à donner la parole - fait trop rare dans la production littéraire et audiovisuelle sur la question - aux conjointes, considérées souvent comme les simples accompagnatrices des migrants. Elles racontent leurs efforts pour s'appro-

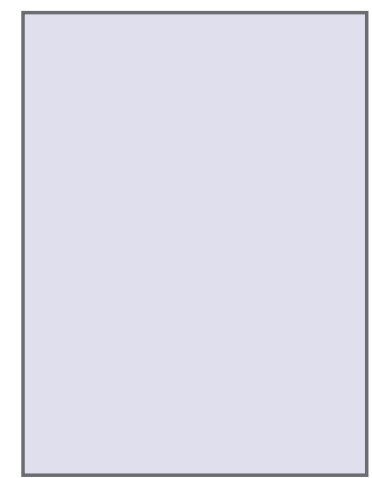

\section{Claudio Giovannesi}

Ali a les yeux bleus Film italien (2012)

Les naufrages d'embarcations de fortuneaularge des côtes italiennes se sont multipliés ces derniers temps. Avec les conflits au Proche et du Moyen-Orient, I'Italie est devenue le théâtre quotidien de ces drames. Étape prier la société d'accueil et leur désir d'y trouver une place. Pour ces femmes qui souvent ne parlaient pas le français, communiquer est une gageure. Maria écrivait sur ses bras les mots entendus sur son lieu de travail pour ne pas les oublier. Madhia s'enregistrait chantant Brel, Bachelet et Piaf dans la caravane qu'elle partageait avec son mari, vendeur itinérant, "pour apprendre la langue".

Aufildecestémoignages, c'està l'histoire sociale de la France que fait écho C'est comme ça. Histoires d'Angevins venus d'ailleurs de 1960 à 1980. Les immigrations auront marqué aussi bien l'action syndicale, que les politiques de la ville et du logement ou les modes de production économique.

Christelle Fifaten Hounsou

1. Didier Fassin, "Introduction. Frontières extérieures, frontières intérieures”, in Didier Fassin (dir.),

Les Nouvelles Frontières de la société française, Paris, La Découverte, 2010.

2. Abdelmalek Sayad, La Double Absence.

Des illusions de l'émigré aux souffrances de l'immigré,

Paris, Seuil, 1999.

obligée de nombreux réfugiés, elle peine à accueillir ces flux de clandestins. Le ministre de l'Intérieur Angelino Alfano a récemment apostrophé les instances européennes à ce sujet et a menacé de laisser partir tous ces demandeurs d'asile, si elles ne venaient pas au secours de l'Italie. La question de l'immigration est sur toutes les lèvres en ce lendemain amer d'élections européennes. Le héros du dernier film du réalisateur italien Claudio Giovannesi n'est pas un primo-arrivant. II fait partie 


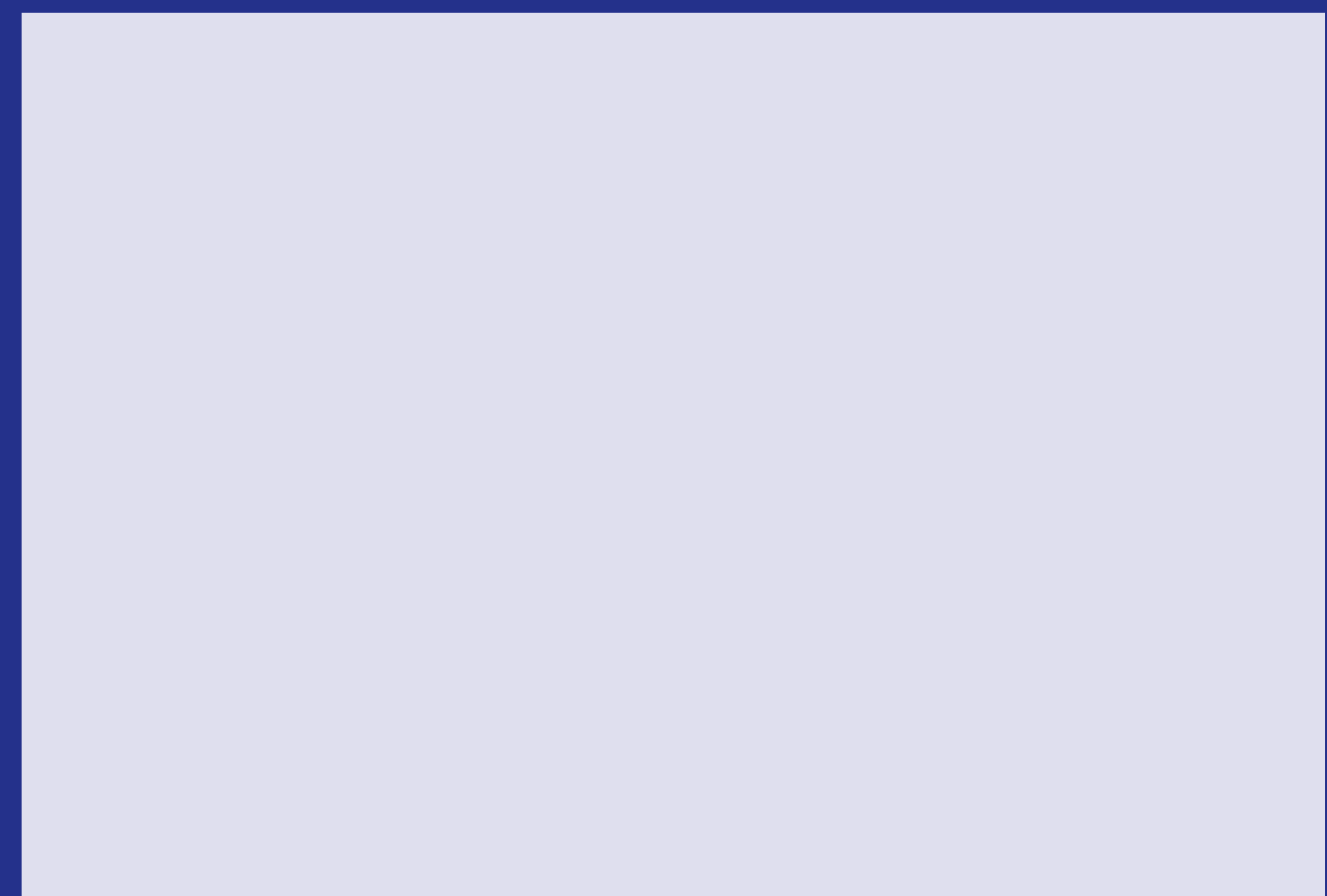

ali a les yeux bleus ( $)$ Photo Nader Sarhan - Bellissima Films

de cette deuxième génération engluée dans une double culture schizophrénique. Il est, d'une part, pétri par l'éducation de ses parents de confession musulmane et, d'autre part, par celle de son pays marqué par le catholicisme. Dans la continuité de son premier documentaire, le cinéaste scrute d'un $œ i l$ bienveillant la jeunesse italienne issue des classes populaires. L'histoire d'amour de Nader, un des acteurs non professionnels de son précédent film - qui joue ici son propre rôle -, est à l'origine de cette fiction. Ostie, banlieue balnéaire de Rome, des adolescents, Nader l'Égyptien d'Ostie, Stefano le Romain, Zoran le Roumain, font les quatre cents coups. Entre cambriolages et petits délits, le quotidien des trois garçons suit son cours. Mais tout bascule lorsque Nader annonce à sa famille sa relation avec Brigitte, une jeune Italienne. Sa mère s'oppose catégoriquement à cette union. Révolté, le jeune homme décide de quitter le cocon familial. Pendant une semaine, on suit l'adolescent tiraillé entre ces deux cultures. Le titre du film est emprunté à un poème de 1962 du sulfureux Pier Paolo Pasolini. Dans une prose prophétique, le cinéaste y parle des rapports Nord/Sud et de la transformation de la société italienne par l'immigration. Ce multiculturalisme est une réalité aujourd'hui. Sur la péninsule, l'immigration représente actuellement pas moins de $10 \%$ de la population, alors que le taux de natalité reste faible. Le film se déroule 
à Ostie et ce n'est pas un hasard. C'est une banlieue multi-ethnique de Rome et c'est sur une plage d'Ostie qu'en 1975 a été retrouvé le corps sans vie de Pier Paolo Pasolini. Nader porte des lentilles bleues, un autre hommage au héros du poème du cinéaste défunt. L'adolescent tente de s'européaniser. En échec scolaire, il ne parvient pas à se conformer à la société catholique. Ayant été luimême élevé dans la religion musulmane, il rejette les symboles chrétiens.

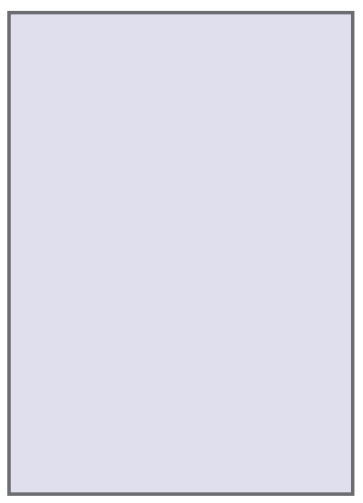

\section{Steve Faigenbaum} City of dream

Documentaire français (2013)

Detroit, État du Michigan, ancien fleuron de l'industrie automobile, est devenu une ville fantôme. Cimetière du rêve américain, la ville incarne la limite du capita-

lisme. Sa faillite progressive jusqu'à la banqueroute a conduit à un exode massif de sa population.

Après vingt-cinq ans d'absence, le cinéaste Steve Faigenbaum revient dans sa ville natale à l'occasion du décès de son père. II y retrouve des cousins, des oncles et tantes et ses souvenirs d'enfance. II raconte la ruine de cette cité.

Se mêlent alors I'histoire personnelle de sa famille d'immigrés juifs polonais et la grande Histoire. De l'apogée au déclin de cet empire, il nous conduit dans le labyrinthe de la mémoire. Images d'archives familiales, publicités,
En cours, il prend à partie son professeur et l'interroge sur le bien-fondé de la présence d'un crucifix sur le mur de la salle de classe. La laïcité italienne est très particulière. La forte prégnance de la la religion dans la société ne facilite pas l'intégration.

Dans une veine naturaliste, le cinéaste dresse un portrait sombre de la société italienne et dénonce l'échec d'une politique d'immigration qui ne parvient pas à insérer ses minorités. Anaïs Vincent

reportages et films institutionnels d'époque illustrent ces réminiscences fragiles. Ses recherches le mènent vers les mouvements migratoires successifs qui peuplèrent et dépeuplèrent cette cité symbole de l'hystérie capitaliste.

Pendant la première moitié du XXe siècle, Detroit, ville industrielle par excellence où prospèrent les trois géants de l'automobile (General Motor, Ford et Chrysler), connait un besoin croissant de main-d'œuvre. En plein essor, elle attire les immigrés européens contraints de fuir la guerre. Les grands-parents du réalisateur, de modestes ouvriers polonais, ont cru aux promesses du rêve américain. Les Noirs des États du Sud sont également attirés par ces nouveaux emplois bien payés. Grâce à cette croissance industrielle, de pauvres ouvriers agricoles peuvent être embauchés dans l'industrie et devenir ainsi propriétaires terriens. Mais, depuis 1945 , avec l'automatisation et les tensions raciales, Detroit n'a cessé de décliner. Les usines se délocalisent dans le sud du pays, le chômage augmente, alors que les immigrés afro-américains conti- 
nuent d'y élire domicile. Les violences interraciales et la ségrégation économique attisent les haines.

En 1967, la révolte des Noirs partie de la $12^{\mathrm{e}}$ rue éclate et durera cinq jours, faisant de nombreux blessés. Ces violences amènent la population blanche à se déplacer vers les banlieues. On parle alors de "white flight". En un demi-siècle, Detroit perd la moitié de sa population. On la surnomme "shrinking city", la ville qui rétrécit?. Les traces des émeutes et les stigmates du chômage l'ont transformée durablement.

Comme beaucoup, Steve Faigenbaum a préféré fuir et reconstruire ailleurs. D'un regard pessimiste, il observe aujourd'hui les ruines de ce passé glorieux. L'espoir d'un avenir radieux s'est évanoui, ne subsistent que les squelettes des maisons abandonnées, vestiges d'un rêve.

Alors qu'il brigue un second mandat aux prochaines élections, le gouverneur républicain du Michigan Rick Snyder

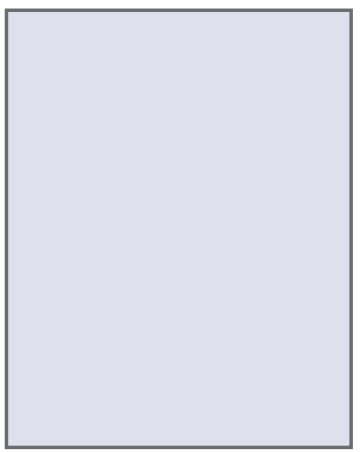

\section{Cherien Dabis May in the summer \\ Film jordanien (2013)}

Après son premier longmétrage très remarqué, Amerrika, la réalisatrice américaine d'origine palestino-jordanienne Cherien Dabis revient avec une jolie fable identitaire sortie le 7 mai dernier sur les écrans parisiens. May, une jeune Jordanienne vivant à New York, vient passer ses vacances porte toute son attention sur la redynamisationdecettevilleportuaire.Iladéclaré en février dernier vouloir faire appel à une immigration hautement qualifiée pour redresser l'économie. II propose de délivrer 500000 visas en cing ans. Cette politique d'immigration choisie à l'échelle municipale pose problème. Comment contraindre ces nouveaux arrivants à rester dans la cité? Ne serontils pas une source de nouvelles tensions au sein de la population ${ }^{2}$ ?

Avec ce documentaire sensible, le cinéaste-archéologue exhume avec délicatesse les fantômes du passé et propose une relecture pertinente de l'effondrement d'un mythe. City of dreams, Detroit une histoire américaine sortira en salle le 9 juillet prochain. A. V.

1. Allan Popelard, Paul Vannier, "Detroit, la ville afroaméricaine qui rétrécit”, in Le Monde diplomatique, janvier 2014

2. "Detroit's Immigration Solution", in The New York Times, 5 février 2014.

d'été à Amman où elle va célébrer son mariage avec un musulman. Sa mère, de confession catholique, ne peut accepter une telle union et fait tout pour dissuader sa fille de se marier. Et May est confrontée à son obstination. En compagnie de ses sœurs, elle entame les préparatifs sur fond d'introspection. Au fil des discussions téléphoniques, sa relation avec Ziad, son futur époux, se fragilise. Avec l'histoire de ce mariage interreligieux, la réalisatrice dépoussière des préjugés bien enracinés. En arrière-plan se dévoile la Jordanie, terre d'asile et d'exil au profil migratoire complexe. 
May in the summer est partiellement autobiographique. La réalisatrice incarne à l'écran son personnage principal. Cherien Dabis est une immigrée de la deuxième génération. Ses parents venus du Moyen-Orient ont immigré au Nebraska, où elle est née. Elle a toujours éprouvé un sentiment de non-appartenance. Elle reste une femme arabe aux États-Unis et une Américaine en Jordanie. May, elle, semble assumer cette double culture jusqu'à l'effritement progressif de ses convictions et de sa recherche identitaire. Dans ce cheminement, elle entraîne le reste de la famille. Sans grandes ressources industrielles et naturelles, la Jordanie, pays à l'économie fragile, a depuis longtemps adopté une politique d'ouverture vers I'Occident. Sous perfusion américaine, elle a signé un accord de libre-échange avec les États-Unis, son principal soutien militaire et économique. Elle demeure pourtant une terre d'exil. Une émigration massive a eu lieu dans les années 1960, conséquence de la flambée du prix du pétrole. Les destinations favorites des émigrés qualifiés jordaniens sont les États-Unis et les Émirats arabes. La Jordanie est aussi une terre d'asile : sa population est composée à $70 \%$ d'habitants d'origine palestinienne. Les conflits limitrophes, avec l'afflux massif de réfugiés irakiens, libanais et palestiniens, et une politique de "porte ouverte" en ont fait une terre d'accueil par excellence. Pendant la guerre du Golfe, la population a augmenté de $10 \%$. Les tensions intercommunautaires se sont intensifiées et le pays a sombré dans la crise'. L'émigration vers les pays du Golfe entre 1973 et 1985 a permis de stabiliser ces tensions politico-économiques. L'État a ainsi pu bénéficier de l'afflux des capitaux des expatriés et de l'aide financière des pays arabes. Avec cette politique, la Jordanie est devenue un État rentier. Mais, avec la crise du Golfe, le retour de ces émigrés et de nouveaux mouvements massifs de déplacement ont mis en péril cette stabilité précaire. En 1994, date du recensement national, les mouvements migratoires vers l'Occident reprennent. En 2008, plus de 280000 Jordaniens vivaient à l'étranger. En 2000, les femmes représentaient $47 \%^{2}$. Elles sont diplômées et très qualifiées. De retour dans son pays d'origine, déracinée, May regarde cette société en pleine mutation avec les yeux d'une intellectuelle occidentale. Jeune femme émancipée, elle exècre les regards inquisiteurs des hommes qui la dévisagent lorsqu'elle fait son footing sur les artères de la ville. Ses sœurs, elles-mêmes ancrées dans la modernité, critiquent avec véhémence les femmes voilées.

La réalisatrice Cherien Dabis nous livre avec ce second long-métrage une chronique familiale enlevée qui flirte tour à tour avec le registre du drame et celui de la comédie. Entre les lignes du scénario transparaît la réalité d'une société complexe marquée par les flux migratoires. Anaïs Vincent

1. Françoise de Bel-Air, "Moyen-Orient : mutations récentes d'un carrefour migratoire, Migrations internationales et politiques en Jordanie", in Revue européenne des migrations internationales, vol 19, $n^{\circ} 3,2003$.

2. Anna di Bartolomeo, Tamirace Fakhoury, Delphine Perrin, "Jordan, the demographic-economic framework of migration, the legal framework of migration, the socio-political framework of migration", in Carim, 2010, www.carim.org. 